\title{
Flavour-symmetry breaking of the quark condensate and chiral corrections to the Gell-Mann-Oakes-Renner relation
}

\author{
Matthias Jaminf \\ Institut für Theoretische Physik, Universität Heidelberg, \\ Philosophenweg 16, D-69120 Heidelberg, Germany
}

(Dated: February 1, 2002)

\begin{abstract}
The relation between the chiral quark condensate in QCD sum rules and chiral perturbation theory is clarified with the help of a low-energy theorem for the scalar and pseudoscalar correlation functions. It is found that the quark condensate should be identified with the non-normal-ordered vacuum expectation value of quark-antiquark fields. Utilising results on flavour SU(3) breaking of the quark condensate from QCD sum rules, the low-energy constant $H_{2}^{r}$ in the chiral Lagrangian, as well as next-to-leading order corrections to the Gell-Mann-Oakes-Renner relation are estimated.

PACS numbers: 12.38.Aw, 11.55.Hx, 12.39.Fe, 11.30.Hv
\end{abstract}

\section{INTRODUCTION}

During the last two decades, QCD sum rules [1] and chiral perturbation theory $(\chi \mathrm{PT})$ [2] have become indispensable tools in the phenomenology of particle physics. Both methods address the problem of dealing with QCD in the non-perturbative low-energy regime. Whereas in QCD sum rules the basic degrees of freedom are quarks and gluons and the non-perturbative region is approached from higher energies by including vacuum averages of composite operators, the condensates, $\chi \mathrm{PT}$ constitutes a low-energy expansion with the lowest lying hadrons as the fundamental fields. Nevertheless, a sound matching of QCD and low-energy effective theories still is an open problem of QCD phenomenology [3].

$\chi \mathrm{PT}$ is constructed such as to reflect the symmetry properties of QCD under chiral transformations. The QCD pattern of explicit chiral symmetry breaking is implemented by adding quark mass terms in the $\chi \mathrm{PT}$ Lagrangian, which introduces an additional free parameter $B_{0}$ at the leading order, besides the pion decay constant $f_{\pi}$. Since the $\chi \mathrm{PT}$ mass term contains the scalar quark density, it turns out that the parameter $B_{0}$ is related to the quark condensate.

The quark condensate in full QCD is not directly related to a physical quantity. Like the QCD coupling and the quark masses, its value depends on the renormalisation scale and on the renormalisation scheme employed in the calculation. 224] On the other hand, in $\chi \mathrm{PT}$ the information on the short-distance properties of the theory is hidden in the low-energy constants, and thus the question arises, how the quark condensates of $\mathrm{QCD}$ and $\chi \mathrm{PT}$ are related. The key to the answer lies in a low-energy theorem for the scalar and pseudoscalar correlation functions, which will be discussed in the next section.

In $\chi \mathrm{PT}$, the dependence of the quark condensate on the renormalisation is reflected in an unphysical low-energy constant $H_{2}^{r}$, which appears at the next-to-leading order and is required for the renormalisation of the chiral expansion. $H_{2}^{r}$ thus comprises information on short- distance physics, which cannot be deduced from $\chi \mathrm{PT}$ alone. In section three, the constant $H_{2}^{r}$ will be estimated from flavour $\mathrm{SU}(3)$ breaking of the quark condensate. This flavour breaking can for example be extracted from QCD sum rules for the heavy-light $B$ - and $B_{s}$-meson systems.

Besides the flavour-symmetry breaking of the quark condensate, $H_{2}^{r}$ also governs the next-to-leading chiral correction to the well known Gell-Mann-Oakes-Renner (GMOR) relation [5. The GMOR relation constitutes the main source for numerical values of the quark condensate, assuming the light quark masses to be known. Making use of the estimate of $H_{2}^{r}$, in section four, also estimates for these chiral corrections and the quark condensate will be presented.

\section{THE SCALAR CORRELATOR}

The central object which is investigated in the seminal version of $\mathrm{QCD}$ sum rules [1] is the two-point function $\Psi\left(p^{2}\right)$ of two hadronic currents

$$
\Psi\left(p^{2}\right) \equiv i \int d x e^{i p x}\left\langle\Omega\left|T\left\{j(x) j(0)^{\dagger}\right\}\right| \Omega\right\rangle,
$$

where $\Omega$ denotes the physical vacuum and in our case $j(x)$ will be the divergence of the flavour-changing vector (axialvector) current,

$$
j(x)=\partial^{\mu}\left(\bar{Q} \gamma_{\mu}\left(\gamma_{5}\right) q\right)(x)=i(M \mp m)\left(\bar{Q}\left(\gamma_{5}\right) q\right)(x) .
$$

$M$ and $m$ represent the masses of the quarks $Q$ and $q$ respectively, and the upper (lower) sign always corresponds to the scalar (pseudoscalar) case. Up to a factor $(M \mp m)^{2}, \Psi\left(p^{2}\right)$ is just given by the scalar (pseudoscalar) two-point function. Furthermore, $\Psi\left(p^{2}\right)$ satisfies a dispersion relation with two subtractions,

$$
\Psi\left(p^{2}\right)=\Psi(0)+p^{2} \Psi^{\prime}(0)+p^{4} \int_{0}^{\infty} \frac{\rho(s)}{s^{2}\left(s-p^{2}-i 0\right)} d s,
$$


where $\rho(s) \equiv \operatorname{Im} \Psi(s+i 0) / \pi$ is the spectral function corresponding to $\Psi(s)$.

A low-energy theorem relates the first subtraction constant $\Psi(0)$ to the product of quark masses and quark condensates:

$$
\Psi(0)=-(M \mp m)(\langle\Omega|\bar{Q} Q| \Omega\rangle \mp\langle\Omega|\bar{q} q| \Omega\rangle) .
$$

The relation (4) can be derived by taking the divergence of the vector (axialvector) correlator, employing current commutation relations in addition [6]. Up to the nextto-leading order in the strong coupling constant, it was also verified in QCD perturbation theory [7].

From the time-ordered product in eq. (11) and Wick's theorem, one naturally obtains normal-ordered condensates. However, in the calculation of $\Psi(0)$ also quartic mass corrections as well as higher-dimensional operators arise. On dimensional grounds, the operator corrections with dimension greater than four are accompanied by inverse powers of the quark masses thus being singular in the chiral limit. If the normal-ordered quark condensates are rewritten in terms of the non-normal-ordered minimally-subtracted condensates, the quartic mass as well as higher-dimensional operator corrections are cancelled, such as to yield the simple functional form of eq. (位) [7, 8, 9, 10].

The fact that the quark condensates in eq. (4) should be considered as non-normal-ordered implies that the subtraction constant $\Psi(0)$ depends on the renormalisation scale and scheme. Whereas the product of quark mass times normal-ordered condensate is a renormalisation invariant quantity [11], this is no longer true for the non-normal-ordered condensate. In the latter case, the renormalisation invariant involves additional quartic mass terms [8]. Numerically, the quartic mass terms only have some relevance for the strange quark. For the lighter up and down quarks they are completely negligible.

By considering QCD sum rules in which the subtraction constant $\Psi(0)$ remains 12, it would in principle be possible to determine the quark condensate or flavourbreaking ratios of condensates. Here, care has to be taken that the quartic mass corrections on the left-hand side of eq. (3), which cancel the renormalisation dependence of $\Psi(0)$ are included properly. Nevertheless, even though the various quantities related to $\Psi(0)$ generally were found to be reasonable, since the sum rules in question are subject to very large higher-order perturbative corrections [13], these results should be interpreted with caution.

\section{THE QUARK CONDENSATE IN $\chi$ PT}

The status of the quark condensate in $\chi \mathrm{PT}$ can be clarified by calculating the scalar (pseudoscalar) correlator and the corresponding low-energy theorem also in this framework [2]. It is then found that the functional form of $\Psi(0)$ exactly resembles eq. (4), and thus the quark condensate in $\chi \mathrm{PT}$ should be identified with the non-normalordered vacuum average of quark-antiquark fields.

As has been already discussed in the introduction, in $\chi \mathrm{PT}$ the quark condensate, as well as flavour-breaking ratios, depend on the unphysical low-energy constant $H_{2}^{r}$. An example of this dependence is displayed by the ratio

$$
\frac{\langle\bar{s} s\rangle}{\langle\bar{q} q\rangle}=1+3 \mu_{\pi}-2 \mu_{K}-\mu_{\eta}+\frac{8 \Delta_{K \pi}}{f_{\pi}^{2}}\left(2 L_{8}^{r}+H_{2}^{r}\right),
$$

where $\langle\bar{q} q\rangle \equiv[\langle\bar{u} u\rangle+\langle\bar{d} d\rangle] / 2$ represents the isospin average of the up- and down-quark condensates, and in the following, the vacuum state $\Omega$ is omitted. Furthermore, the $\mu_{P}$ are chiral logarithms which take the form

$$
\mu_{P}=\frac{M_{P}^{2}}{32 \pi^{2} f_{\pi}^{2}} \ln \frac{M_{P}^{2}}{\nu_{\chi}^{2}},
$$

with $\nu_{\chi}$ being the chiral renormalisation scale, $\Delta_{K \pi}=$ $M_{K}^{2}-M_{\pi}^{2}$, and $L_{8}^{r}$ is a physical low-energy constant in the next-to-leading order chiral Lagrangian $[2$.

The relation (5) offers the possibility to determine the low-energy constant $H_{2}^{r}$, since the ratio $\langle\bar{s} s\rangle /\langle\bar{q} q\rangle$ can be obtained independently, e.g. in the framework of QCD sum rules. A rather reliable place to determine $\langle\bar{s} s\rangle /\langle\bar{q} q\rangle$ are sum rules for the leptonic decay constants of the heavy $B$ - and $B_{s}$-mesons 14 (and references therein), since the deviation of the flavour $\mathrm{SU}(3)$ breaking ratio $f_{B_{s}} / f_{B}$ from one is very sensitive to the quark condensate ratio $\langle\bar{s} s\rangle /\langle\bar{q} q\rangle$.

The ratio of the heavy meson decay constants $f_{B_{s}} / f_{B}$ is, unfortunately, not yet known experimentally. Thus we have to resort to a different framework to find results on this ratio, independent of QCD sum rules. Such results have been obtained in the framework of lattice QCD and a recent average yielded $f_{B_{s}} / f_{B}=1.16 \pm 0.04$ [15]. 25] Employing this value in the QCD sum rules, the corresponding flavour breaking ratio of the quark condensates turns out to be

$$
\langle\bar{s} s\rangle /\langle\bar{q} q\rangle=0.8 \pm 0.3 .
$$

Within the uncertainties, this result is in agreement to previous determinations 17]. Nevertheless, older results have not been included in eq. (7), since in these investigations normal-ordered condensates have been used and the corresponding quark mass corrections have not been included. A proper treatment of the determination of $\langle\bar{s} s\rangle /\langle\bar{q} q\rangle$ from QCD sum rules for other channels will be left for future work.

In addition to the ratio $\langle\bar{s} s\rangle /\langle\bar{q} q\rangle$, for the determination of $H_{2}^{r}$ also the low-energy constant $L_{8}^{r}$ is required. This constant can be determined from flavour-symmetry breaking of meson masses and decay constants, which at 
next-to-leading order in $\chi \mathrm{PT}$ satisfies the relation [2]

$\frac{1}{(R+1)} \frac{M_{K}^{2}}{M_{\pi}^{2}}+\frac{f_{K}}{f_{\pi}}=\frac{3}{2}+\frac{3}{4} \mu_{\pi}-\frac{1}{2} \mu_{K}-\frac{1}{4} \mu_{\eta}+\frac{8 \Delta_{K \pi}}{f_{\pi}^{2}} L_{8}^{r}$.

Here $R$ represents the quark-mass ratio $R \equiv m_{s} / \hat{m}=$ $24.4 \pm 1.5$ [18, and the value $f_{K} / f_{\pi}=1.22 \pm 0.01$ [19] will be used. Employing the relation (8), the constant $L_{8}^{r}$ is found to be

$$
L_{8}^{r}\left(\nu_{\chi}=M_{\rho}\right)=(0.88 \pm 0.24) \cdot 10^{-3},
$$

where the pion decay constant has been varied in the range $f_{\pi}=92.4 \pm 10.0 \mathrm{MeV}$. Although the experimental uncertainty for $f_{\pi}$ is much smaller [19], the chosen error reflects the difference between the physical value and the leading order decay constant $f_{0}=82 \mathrm{MeV}$, such that uncertainties from higher orders in the chiral expansion should be largely taken into account. The result (9) is in agreement to the original value obtained by Gasser and Leutwyler [2] at the scale $\nu_{\chi}=M_{\eta}$.

The finding of eq. (9) for $L_{8}^{r}$ can also be compared with the very recent value $L_{8}^{r}\left(\nu_{\chi}=M_{\rho}\right)=(0.62 \pm 0.20) \cdot 10^{-3}$ [20], which has been obtained in $\chi \mathrm{PT}$ at $\mathcal{O}\left(p^{6}\right)$. Within the uncertainties both results are compatible, but it is seen that including the next-next-to-leading corrections in the chiral expansion effectively lowers the constant $L_{8}^{r}$. Since the presented analysis stays at the next-to-leading order, for consistency also the corresponding value (9) for $L_{8}^{r}$ has been utilised in the following.

Inserting the results of eqs. (7) and (9) into (5), it is possible to deduce an estimate on the unphysical lowenergy constant $H_{2}^{r}$ :

$$
H_{2}^{r}\left(\nu_{\chi}=M_{\rho}\right)=(-3.4 \pm 1.5) \cdot 10^{-3} .
$$

The negative value for $H_{2}^{r}\left(\nu_{\chi}=M_{\rho}\right)$, found in eq. (10), is in contrast to estimates of the same quantity in the framework of $\chi \mathrm{PT}$ with explicit inclusion of resonance fields [21]. Assuming saturation of the low-energy constants in the chiral Lagrangian by meson resonances, reasonable values for the physical constants $L_{i}^{r}$ are obtained, whereas in the case of $H_{2}^{r}$, saturation with scalar resonances yields the relation $H_{2}^{r}=2 L_{8}^{r}$, which is clearly violated by the result (10). 26] Since $H_{2}^{r}$ and $2 L_{8}^{r}$ have the same dependence on the chiral scale $\nu_{\chi}$, it is even impossible to find a particular scale at which the relation $H_{2}^{r}=2 L_{8}^{r}$ would hold.

\section{CHIRAL CORRECTIONS TO THE GELL-MANN-OAKES-RENNER RELATION}

The unphysical low-energy constant $H_{2}^{r}$ also appears in the next-to-leading order chiral corrections to the GMOR relations [2, 5],

$$
\begin{aligned}
4 \hat{m}\langle\bar{q} q\rangle & =-2 f_{\pi}^{2} M_{\pi}^{2}\left(1-\delta_{\pi}\right), \\
\left(m_{s}+\hat{m}\right)[\langle\bar{s} s\rangle+\langle\bar{q} q\rangle] & =-2 f_{K}^{2} M_{K}^{2}\left(1-\delta_{K}\right),
\end{aligned}
$$

where $\hat{m} \equiv\left(m_{u}+m_{d}\right) / 2$ is the isospin average of the light up- and down-quark masses, and $\delta_{\pi}$ as well as $\delta_{K}$ comprise the higher-order chiral corrections. The lefthand sides of eqs. (11) and (12) just correspond to minus $\Psi(0)$ in the pseudoscalar case with $(u d)$ and $(s q)$ quark flavours respectively. At the next-to-leading order, these corrections have been found to be [2]:

$$
\delta_{\pi}=4 \frac{M_{\pi}^{2}}{f_{\pi}^{2}}\left(2 L_{8}^{r}-H_{2}^{r}\right) \quad \text { and } \quad \delta_{K}=\frac{M_{K}^{2}}{M_{\pi}^{2}} \delta_{\pi} .
$$

The results for $H_{2}^{r}$ and $L_{8}^{r}$, obtained in the last section, now allow for an estimation of the chiral corrections $\delta_{\pi}$ and $\delta_{K}$, which take the values

$$
\delta_{\pi}=0.047 \pm 0.017 \quad \text { and } \quad \delta_{K}=0.61 \pm 0.22 .
$$

With roughly $5 \%$, the size of the correction $\delta_{\pi}$ appears very reasonable, whereas $\delta_{K}$ in the strange quark sector turns out rather large, due to the enhancement factor $M_{K}^{2} / M_{\pi}^{2}$, although the uncertainties are also quite big. If, on the other hand, the relation $H_{2}^{r}=2 L_{8}^{r}$ which follows from scalar resonance saturation 21] would be correct, both chiral corrections $\delta_{\pi}$ and $\delta_{K}$ would have to vanish.

The knowledge of the light quark masses also allows for a determination of the light quark condensate from the first GMOR relation (11). Using the recent result $\left(m_{u}+m_{d}\right)(2 \mathrm{GeV})=8.1 \pm 1.4 \mathrm{MeV}[13$, together with the estimate (14) for $\delta_{\pi}$, one arrives at

$$
\langle\Omega|\bar{q} q| \Omega\rangle(2 \mathrm{GeV})=-(267 \pm 16 \mathrm{MeV})^{3},
$$

which can be considered as an update of previous determinations of the light-quark condensate $\langle\bar{q} q\rangle$. Since it is still more common to quote the quark condensate at a scale of $1 \mathrm{GeV}$ the corresponding value $\langle\bar{q} q\rangle(1 \mathrm{GeV})=$ $-(242 \pm 15 \mathrm{MeV})^{3}$ is also provided. The latter results are in good agreement with direct determinations of $\langle\bar{q} q\rangle$ from QCD sum rules [22] and lattice QCD [23].

\section{CONCLUSIONS}

From the structure of the low-energy theorem of eq. (4) for the scalar (pseudoscalar) two-point function, it is possible to infer that the quark condensate in $\chi \mathrm{PT}$ should be identified with the non-normal-ordered vacuum average of quark-antiquark fields. Contrary to the corresponding case of normal-ordered condensates, the product of quark mass times non-normal-ordered condensate, and thus $\Psi(0)$, are not renormalisation invariant. In $\chi \mathrm{PT}$ 
the dependence of $\Psi(0)$ on the short-distance renormalisation scale and scheme is reflected in the dependence on the unphysical low-energy constant $H_{2}^{r}$.

Because the low-energy constant $H_{2}^{r}$ depends on the renormalisation at short-distances, its value cannot be deduced from relations to physical quantities alone. One possible quantity to determine $H_{2}^{r}$ is the $\mathrm{SU}(3)$ flavourbreaking ratio of the quark condensates $\langle\bar{s} s\rangle /\langle\bar{q} q\rangle$. The estimate presented in eq. (10) has been obtained on the basis of $\langle\bar{s} s\rangle /\langle\bar{q} q\rangle$ from QCD sum rules for the heavy $B$ and $B_{s}$-mesons, making additional use of results on the ratio $f_{B_{s}} / f_{B}$ from lattice $\mathrm{QCD}$.

The obtained value for $H_{2}^{r}$ also allows to estimate the chiral corrections to the GMOR relations of eqs. (11) and (12). It is found that the first GMOR relation in the light up- and down-quark sector receives a moderate negative correction of the order of $5 \%$, whereas the corresponding correction in the strange-quark sector is enhanced by a factor $M_{K}^{2} / M_{\pi}^{2}$, and thus turns out to be rather large. Together with values for the light quark masses, the first GMOR relation makes it possible to determine the light quark condensate $\langle\bar{q} q\rangle$, and an updated value has been presented in the last section.

Since the low-energy constants of $\chi \mathrm{PT}$ contain the physics of energies higher than the pseudoscalar meson masses, it is possible to derive effective Lagrangians including higher resonance fields, which then yield contributions to the chiral constants 21. For the physical constants $L_{i}^{r}$, very reasonable estimates were obtained in this manner. For the unphysical constant $H_{2}^{r}$, however, it was shown above that the result $H_{2}^{r}=2 L_{8}^{r}$ from the resonance Lagrangian is not satisfied. It should already be clear that this relation has to receive additional contributions, since $H_{2}^{r}$ depends on the short-distance renormalisation, whereas this is not the case for $L_{8}^{r}$.

Nevertheless, the uncertainties in the ratio $\langle\bar{s} s\rangle /\langle\bar{q} q\rangle$ are still sizeable, so that no definite conclusions can be reached at this stage. Further work should be devoted to the extraction of $\langle\bar{s} s\rangle /\langle\bar{q} q\rangle$ from QCD sum rules, particularly paying attention to the proper definition of the quark condensate as well as to the corresponding higherorder strange-mass corrections. In addition, an analysis analogous to the one presented here at the next-nextto-leading $\mathcal{O}\left(p^{6}\right)$ in $\chi \mathrm{PT}$ could provide further useful information on the questions raised above, since also the uncertainty resulting from these higher orders plays an important role.

It is a great pleasure to thank J. Prades, H. G. Dosch, A. Pich and E. de Rafael for helpful discussions. The author would also like to thank the Deutsche Forschungsgemeinschaft for their support.

* Electronic address: amin@uni-hd.de; Heisenberg fellow.
[1] M.A. Shifman, A.I. Vainshtein, and V.I. Zakharov, Nucl. Phys. B147, 385, 448 (1979).

[2] J. Gasser and H. Leutwyler, Ann. Phys. 158, 142 (1984); Nucl. Phys. B250, 465, 517, 539 (1985).

[3] S. Peris, M. Perrottet, and E. de Rafael, JHEP 05, 011 (1998).

[4] M. Beneke, Phys. Rep. 317, 1 (1999).

[5] M. Gell-Mann, R.J. Oakes, and B. Renner, Phys. Rev. 175, 2195 (1968).

[6] D.J. Broadhurst, Nucl. Phys. B85, 189 (1975).

[7] D.J. Broadhurst, Phys. Lett. 101B, 423 (1981).

[8] V.P. Spiridonov and K.G. Chetyrkin, Sov. J. Nucl. Phys. 47, 522 (1988).

[9] M. Jamin and M. Münz, Z. Phys. C60, 569 (1993).

[10] C.A. Dominguez et al., Phys. Rev. D51, 5090 (1995).

[11] J.C. Collins, A. Duncan, and S.D. Joglekar, Phys. Rev. D16, 438 (1977); R. Tarrach, Nucl. Phys. B196, 45 (1982).

[12] V.A. Novikov et al., Nucl. Phys. B191, 301 (1981); S. Narison, Phys. Lett. 104B, 485 (1981); S. Narison, N. Paver, and D. Treleani, Nuov. Cim. 74A, 347 (1983); C.A. Dominguez et al., Z. Phys. C27, 481 (1985); C.A. Dominguez and M. Loewe, Phys. Rev. D31, 2930 (1985); C.A. Dominguez and E. de Rafael, Ann. Phys. 174, 372 (1987); S. Narison, Riv. Nuovo Cim. 10N2, 1 (1987); S. Narison, Phys. Lett. B216, 191 (1989); J. Bijnens, J. Prades, and E. de Rafael, Phys. Lett. B348, 226 (1995); S. Narison, Phys. Lett. B358, 113 (1995); C.A. Dominguez, A. Ramlakan, and K. Schilcher, Phys. Lett. B511, 59 (2001).

[13] M. Jamin, J.A. Oller, and A. Pich, hep-ph/0110194.

[14] M. Jamin and B.O. Lange, Phys. Rev. D65, 056005 (2002).

[15] C. Bernard, Nucl. Phys. Proc. Suppl. 94, 159 (2001).

[16] A. Ali Khan et al. . Phys. Rev. D64, 054504 (2001); C. Bernard et al., hep-lat/0110072; N. Yamada et al., hep-lat/0110087.

[17] B.L. Ioffe, Nucl. Phys. B188, 317 (1981); Err.-ibid. B191, 591 (1981); D. Espriu, P. Pascual, and R. Tarrach, Nucl. Phys. B214, 285 (1983); H.G. Dosch, M. Jamin, and S. Narison, Phys. Lett. B220, 251 (1989); K. Langfeld and M. Schaden, Phys. Lett. B272, 100 (1991).

[18] H. Leutwyler, Phys. Lett. B378, 313 (1996).

[19] Particle Data Group, D.E. Groom et al., Eur. Phys. J. C15, 1 (2000).

[20] G. Amoros, J. Bijnens, and P. Talavera, Nucl. Phys. B602, 87 (2001).

[21] G. Ecker et al., Nucl. Phys. B321, 311 (1989).

[22] H.G. Dosch and S. Narison, Phys. Lett. B417, 173 (1998).

[23] L. Giusti et al., Nucl. Phys. B538, 249 (1999); Phys. Rev. D64, 114508 (2001); P. Hernández et al., Nucl. Phys. Proc. Suppl. 106, 766 (2002).

[24] Contrary to the gluon condensate, the quark condensate, being an order parameter of chiral symmetry breaking, does not suffer from a renormalon ambiguity 4 .

[25] More recent results can be found in refs. [16].

[26] In fact, employing the relation $H_{2}^{r}=2 L_{8}^{r}$, lead the authors of 20] to deduce $\langle\bar{s} s\rangle /\langle\bar{q} q\rangle=1.69$. 